\title{
SUPERCOMPUTER APPLICATIONS IN COMPUTATIONAL FLUID DYNAMICS
}

\author{
Terry L. Holst
}

Chief, Applied Computational Fluids Branch NASA Ames Research Center, Moffett Field, California

\begin{abstract}
The field of Computational Fluid Dynamics (CFD) involving largescale supercomputer applications is discussed. Formulational and computational requirements for the various governing equations, including the Euler and Navier-Stokes approaches, are examined for typical problems including the viscous flow field solution about a complete aerospace vehicle. Recent computed results and experimental comparisons are given to highlight the presentation. The future of CFD associated with three-dimensional applications is found to be rapidly expanding across a broad front including internal and external flows, and flows across the entire speed regime.
\end{abstract}

\section{INTRODUCTION}

The fields of Computational Fluid Dynamics (CFD) and computer hardware design have been rapidly advancing over the last decade. It is now possible to demonstrate the importance of CFD in the field of aircraft design and performance prediction for general flow conditions including reasonably complete viscous effects. The problem of solving the nonlinear governing system of partial differential equations, the Euler and Navier-Stokes equations, about complex three-dimensional geometries has been undertaken and significant results have already been produced. The purpose of this paper is to describe the current status of CFD applications in this area and to indicate prospects for the future, especially for supercomputer applications involving complex geometries. Computational requirements for producing these results both now and in the future will be addressed.

This paper is divided into several sections as follows: CFD Formulations, where the major governing equations used in CFD are described with special emphasis on the Euler and NavierStokes formulations; Example Solutions, including two external and one internal applications; and Concluding Remarks. The first item for discussion is how the various CFD formulations, and in particular, those formulations requiring supercomputers for typical applications, fit into the general CFD hierarchical scheme.

\section{CFD FORMULATIONS}

The aforementioned improvements in computer execution speed and main memory capacity achieved in the past several years has made it possible to dramatically expand the field of CFD. This has resulted in significant progress up the hierarchal "ladder" of CFD formulation complexity as shown in Fig. 1. Panel method solutions, which effectively solve the linear Laplace equation using the principle of superposition, have been studied thoroughly during the past three decades. As a result, panel method codes are well established within industry as efficient and reliable design tools for aircraft in the subsonic and supersonic speed regimes.

The next rung on the CFD formulational ladder is occupied by nonlinear potential formulations including the transonic small-disturbance and full potential equations. Both of these formulations have been extensively studied for the past 15 years and except for some grid generation limitations associated with complex geometry applications, these methods are well developed and heavily utilized within the aircraft industry design environment. These nonlinear potential methods extend the range of applicability of CFD to the transonic speed regime and

U.S. Government Work. Not protected by 51

U.S. copyright. 


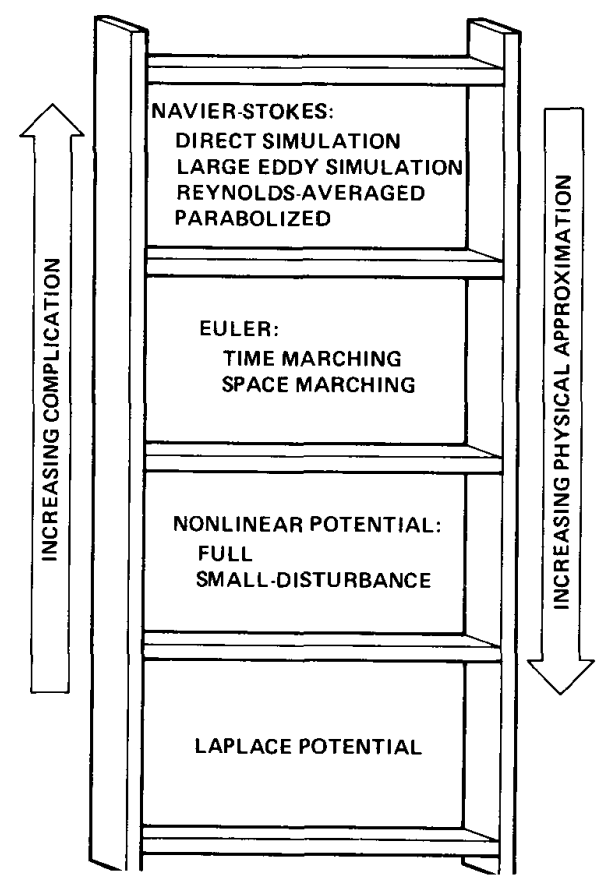

Figure 1. Computational Fluid Dynamics formulational ladder.

therefore have the capability of capturing weak shock waves and wave-drag levels. An appropriate review of this area can be found in [1].

The Euler equation formulation, which occupies the next rung on the CFD formulational ladder, is currently at the center of research in the Aerospace CFD community. New algorithms and applications in three dimensions have proliferated at a very fast rate in the past five years. The various design groups within industry have already started using Euler codes for many applications but significant improvements in code and/or computer efficiency have to be realized before the Euler formulation will be used extensively. The Euler formulation provides better accuracy for strong shock calculations and has the ability to properly track vorticity gradients. These features are important for a variety of advanced flow field situations including vortical aerodynamic studies, strong shock calculations, high angle-of-attack leading-edge flow separation, high-speed blunt body problems, and blast wave studies.

The highest entry on the CFD formulational ladder is occupied by the Navier-Stokes equations. This formulation is the most complex and represents the smallest level of physical approximation. Like the Euler formulation, much work has been completed in the threedimensional Navier-Stokes area during the past five years. However, the application of typical Navier-Stokes codes in the industry design environment has been slow to evolve. This is largely due to two reasons: First, the Navier-Stokes equations are several times more expensive to solve (at least) than the Euler equations. This is due to more terms in the Navier-Stokes equations; more grid points required to resolve a given problem resulting from viscous effects; and in separated flow cases, more iterations are required to achieve convergence because of the more complicated flow field physics. The second major reason that the Navier-Stokes equations have not been used in design applications is due to the complications of turbulence. To discuss this subject a further breakdown of the Navier-Stokes formulation is required. 
The Navier-Stokes equations describe the motion of most fluid flow phenomena including large scale motions associated with the inviscid features of a flow field and small scale motions associated with turbulence. The numerical procedure which directly solves the Navier-Stokes equations and resolves all flow length scales including the smallest turbulent length scales is called direct simulation (DS). When the numerical grid resolution is not fine enough to resolve all length scales problems occur. Because for high Reynolds number flow the range of length scales is so immense, it is not currently possible to simulate this type of flow with schemes based on the DS approach. Thus, the first level of approximation to the Navier-Stokes equations, called Large Eddy Simulation (LES), is introduced. In the LES formulation the Navier-Stokes equations are solved on a grid capable of resolving all length scales except the smallest turbulent length scales, which are associated with the smallest turbulent eddies. Because these smallest eddies tend to be isotropic, they are easier to model than the larger eddies which tend to be random and can vary in nature for different flow conditions. A good review of the DS and LES areas is given in [2].

The LES formulation is still quite expensive and is restricted to problems with limited geometrical complexity and to moderate and low Reynolds numbers. This leads to the Reynolds-averaged Navier-Stokes (RANS) formulation where all turbulent eddies are modeled. The RANS formulation is derived by decomposing all dependent variables, e.g., density, pressure, and velocity components, into two parts, a mean part and an unsteady part. The unsteady part contains the unsteadiness due to high-frequency turbulent fluctuations and the mean part contains the average local flow value for that variable including any low-frequency time variation. After this variable decomposition process the resulting Navier-Stokes equations are time averaged over a time scale which is large in comparison with turbulent fluctuations but small in comparison with mean flow field unsteadiness. As a result of the time-averaging process, certain new terms arising from turbulent eddy correlations remain. These new terms account for the mean or average effect of turbulence and must be modeled. This allows the numerical solution of the Navier-Stokes equations for many applications involving realistic Reynolds numbers on grids that will fit on existing supercomputers.

A comparison of the estimated memory and execution time requirements for several different formulations is given in Fig. 2. An additional horizontal scale indicates the amount of execution time required by a theoretical gigaflop computer, which should be available in the near-term future. The Euler and Navier-Stokes formulations just discussed (DS, LES, and RANS) are all included. Note that several variations of the RANS formulation (steady ideal gas, steady nonequilibrium real gas, and unsteady ideal gas) are presented. Including nonequilibrium real gas effects associated with hypersonic flight or the unsteadiness effects associated with (for example) flight at high angles of attack, may increase the computational costs of a typical steady RANS calculation by an order of magnitude. This additional expense while prohibitive today may not be prohibitive in the near-term future. Additional discussion on the estimated computational costs for various CFD applications can be found in $[3,4]$.

The prospects of computing three-dimensional flows at realistic flight Reynolds numbers using the LES formulation in the near future do not seem bright. As indicated in Fig. 2 the cpu time for such a calculation on a theoretical gigaflop computer would be on the order of one month. The DS picture is even more dismal with cpu times for a single three-dimensional calculation requiring on the order of years. In addition, applications associated with mean flow unsteadiness, real gas effects, or even particularly large Reynolds numbers, could increase these times by orders of magnitude. Hence, it seems that the most appropriate Navier-Stokes formulation for attention in the area of applications involving realistic flight conditions and reasonably complex geometries, is the RANS formulation. The remainder of this paper will describe several CFD applications utilizing the RANS formulation for reasonably complete geometric configurations. For an indepth technical discussion of most of the CFD formulations just discussed, the interested reader is referred to [5]. 


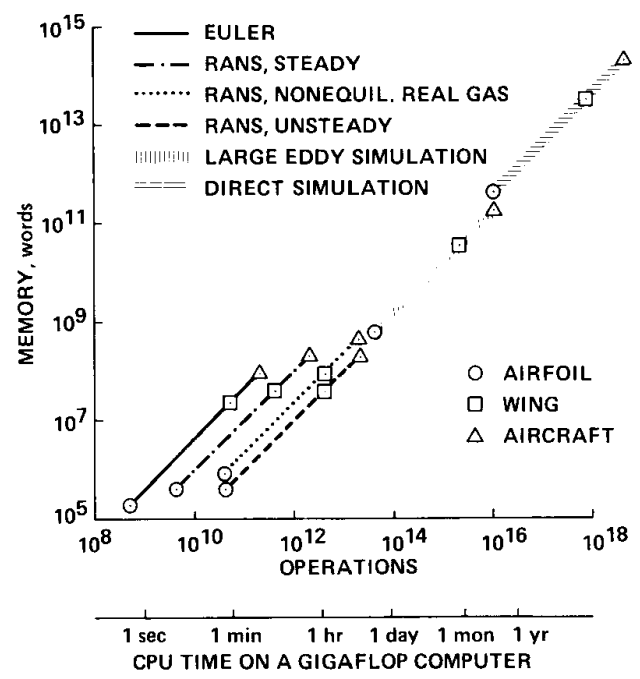

Figure 2. Estimated memory and execution time requirements for several different CFD formulations.

\section{EXAMPLE RANS APPLICATIONS}

The first pioneering CFD calculations with the Navier-Stokes formulation started appearing in the early 70 s. These first of a kind demonstration calculations were severely limited by computational resources available in this time period, both execution speed and memory. Calculations were generally limited to two spatial dimensions and involved simple geometric shapes, e. g., flat plates, circular cylinders, wedges, or perhaps at most, airfoils. Solutions generally involved turbulent flows with simple algebraic turbulence models (or laminar solutions with no turbulence models). These aspects were required to eliminate the complexity of turbulence and to reduce computational requirements. A good review of early work in this area is given by Peyret and Viviand [6].

Several years after the first two-dimensional Navier-Stokes solutions appeared, threedimensional simulations were presented, again using algebraic turbulence models (or a laminar flow assumption) and simple geometries. Since then improvements in computers and algorithms have allowed tremendous advances in CFD across a broad front of different applications. These areas are reviewed in two interesting articles by Chapman $[7,8]$ where the entire field of Computational Aerodynamics is reviewed. Pacing items including turbulence modeling, three-dimensional grid generation, algorithm development, and computer speed limitations are discussed; and computational requirements and projected computer capabilities are examined. More recent surveys are presented by Kutler [9] where the same critical pacing items are examined and updated and by Kutler et al. [10] where CFD methods, applications and computer technology are reviewed. To illustrate current CFD capabilities, several recent examples utilizing the RANS formulation are now presented and discussed.

\section{F-16 Simulation}

The first example of state-of-the-art RANS technology in a practical application is the numerical simulation of the flow about a complete F-16A aircraft. This was accomplished with a series of flow solutions about several increasingly complete geometrical representations of the F-16A. 
This series started with a simple wing-fuselage configuration presented by Reznick and Flores [11] and Flores et al. [12], proceeded with a wing-fuselage-inlet configuration presented by Flores et al. [13], and concluded with the full configuration by Flores and Chaderjian [14]. The geometry in the final simulation consisted of wings, fuselage, inlet, vertical and horizontal tails, and nozzle, and included both sides of the aircraft, that is, sideslip conditions were possible.

This level of geometric complexity was possible because of the zonal grid scheme used in the simulation. The flow field domain was broken into a series of different grid zones, as many as 54 for the sideslip case. One iteration of the solution procedure was performed in each zone followed by appropriate boundary condition implementation. Once all interior and boundary points of each zone were updated, the solution procedure continued to the next iteration until convergence was reached after approximately 2000-5000 iterations. This required about 20-50 hours of cpu time on a Cray XMP/48 computer using a single processor. The sideslip case grid consisted of about one million points and therefore had a total solution database of about eight million words, five dependent variables and the three spatial locations for each grid point. Because of the zonal philosophy and the fact that only one zone was in main memory at a time, the main-memory run-time requirement was under two million words. Without the zonal approach, the run-time memory requirement would have been over 20 million words.

Results from Flores and Chaderiian [14] are presented in Fig. 3 which shows surface pressure contours over the F-16A aircraft for transonic flow conditions at an angle of attack of $6 \mathrm{deg}$ and no sideslip. For this calculation the Baldwin-Lomax [15] tubrulence model was used to simulate turbulent boundary layers on all aircraft surfaces. The jet exhaust is displayed as temperature contours in the jet symmetry plane. The surface pressure contour pattern clearly shows a "lamda" shock system on the upper wing surface, which is typical of transonic sweptwing flow fields. Figure 4 shows comparisons between computed and measured wing surface pressure distributions. For this computation a 0.02 correction in the freestream Mach number was used to improve the comparison [16]. This correction is required to help account for wind tunnel wall effects since the computation was made in free air. Agreement is generally good with the largest disagreement ocurring in the aft shock location near the tip on the wing upper surface.

\section{Space Shuttle Simulation}

The next example of RANS technology is the numerical solution of flow about the Space Shuttle in ascent or launch mode [17]. In this simulation the Orbiter, External Tank (ET), and Solid Rocket Boosters (SRB) are all simulated. This represents a different type of simulation difficulty, that associated with multiple, interferring bodies. The RANS equations, with Baldwin-Lomax turbulence model [15], were solved for this configuration using a special type of zonal grid scheme called the Chimera grid scheme. In this approach the grids about each separate body are generated essentially without regard for the other bodies in the flow field. Solutions are obtained, as in the previous case, by completing one iteration in each grid zone followed by boundary condition updates. The interface conditions between the various grid zones are more complicated in this case, relative to the previous one, and involve a general three-dimensional interpolation procedure.

Results from this study are shown in Fig. 5 for a freestream Mach number of 1.05 and an angle of attack of $-3 \mathrm{deg}$. This set of conditions is near the maximum dynamic pressure point in the Space Shuttle launch trajectory. A comparison of computed and wind tunnel surface pressures is presented for the entire configuration looking down on the orbiter. The computed results are displayed in the upper portion of the figure and the wind tunnel results in the lower portion. Note the generally good agreement even though the computation was performed with stings emanating from the aft ends of the orbiter and SRB, while the wind tunnel test had stings emanating from only the SRBs (not shown). This difference was largely motivated by computational convenience and will be removed in future calculations. Other discrepancies are caused by inaccuracies in modelling the attach hardware between the orbiter and the ET, wind tunnel wall interference, other small protuberances, and lack of sufficient grid resoultion. 


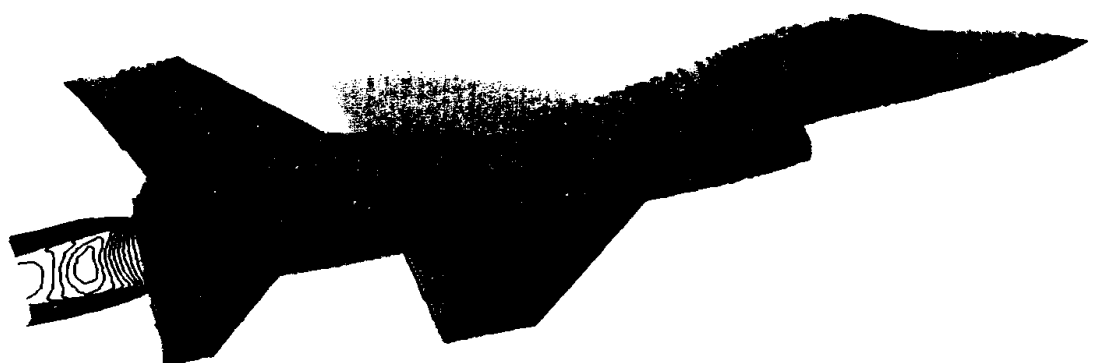

Figure 3. Computed surface pressure contours on the F-16A aircraft and temperature contours along the jet synmmetry plane, $\mathrm{Mach}=0.9, \mathrm{Re}=4.5$ million, Flores and Chaderjian [14].

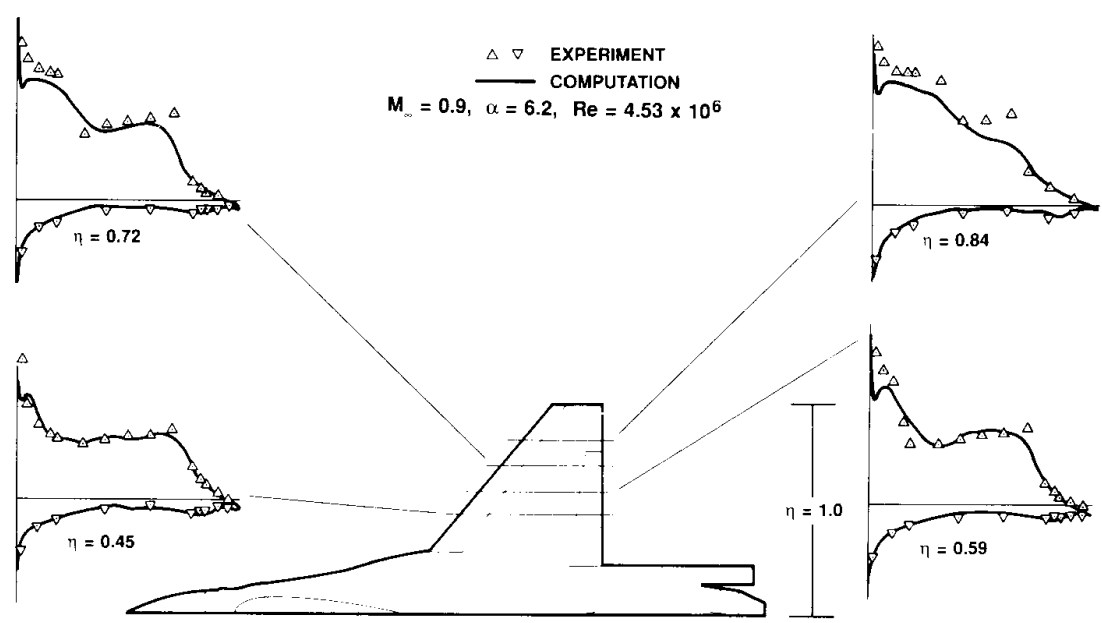

Figure 4. Pressure coefficient comparisons on the F-16A aircraft wing, Mach $=0.9, \mathrm{Re}=$ 4.5 million, Chaderjian and Flores [16].

Figure 6 shows a more quantitative comparison of pressures along the side of the orbiter, indicated by the dashed line. Three sets of results are displayed for two different Mach numbers (1.05 and 0.9) and include computation, wind tunnel, and flight test. The computational results are generally in good agreement with experiment and show smaller disagreements with the two different types of measurements than the disagreements between the two measurements. The largest experimental/computational diagreement appears near the aft portion of the vehicle for the 0.9 Mach number case. This is caused by an inboard-wing control surface deflection, which is included in both the flight and wind tunnel measurements but not in the computation. The deflected control surface is included in the 1.05 Mach number computation, and the three results are in excellent agreement in this vicinity. Thus, CFD simulations are capable of quantitatively discerning relatively small, geometrically-induced flow field perturbations.

The total computer time required for this computation was about 20 hours on a Cray 2 supercomputer using a single processor. The grid consisted of five grid zones and about 750,000 points, which resulted in a total data base of about six million words. 


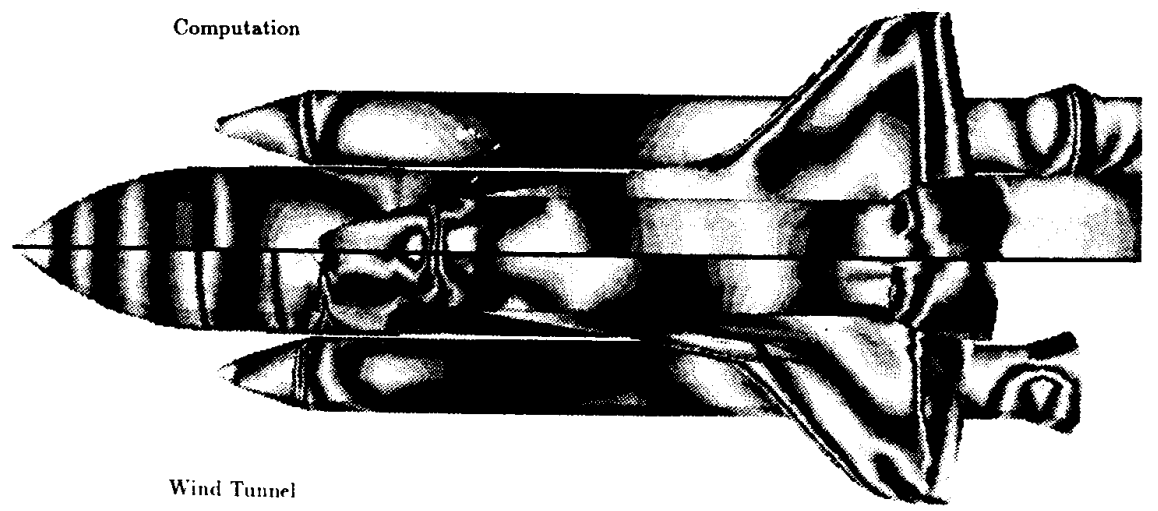

Figure 5. Comparison of pressure coefficient between computation and wind tunnel, Mach $=$ $1.05, \mathrm{Re} / \mathrm{ft}=4$ million (3\% model), [17].
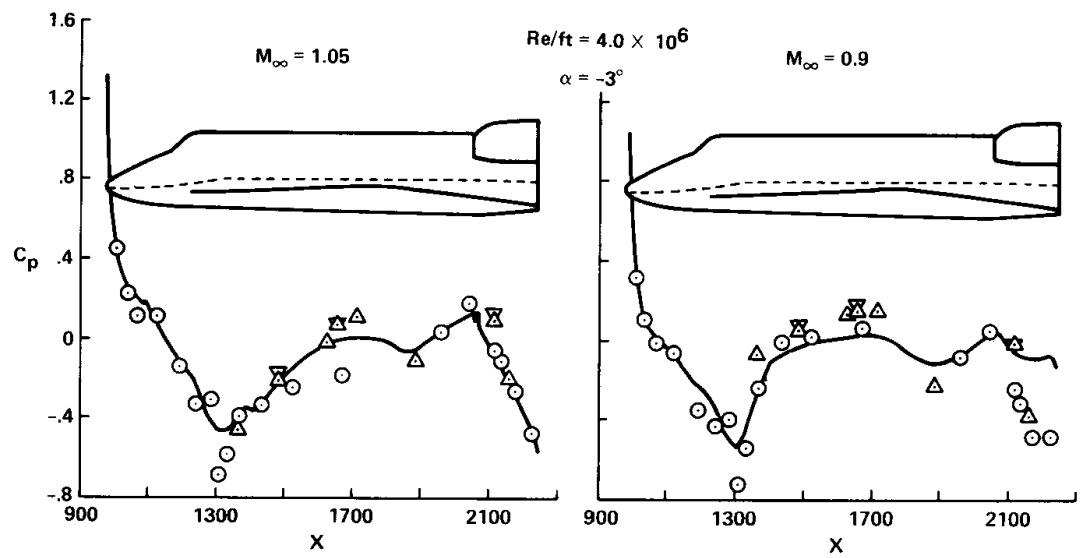

Figure 6. Comparison of pressure coefficient along the Orbiter fuselage just above the wing during Space Shuttle ascent, computation (solid lines), wind tunnel (circular symbols), and flight (triangular symbols), [17].

\section{Turbomachinery Flow Simulation}

The last example of recent RANS simulation technology is associated with an internal flow application, in particular, a turbojet engine. Within all turbojet engines are compressors and turbines composed of alternating rows of fixed or "stator" blades and rotating or "rotor" blades. The function of the compressor is to compress the incoming air for optimum combustion, and the function of the turbine is to extract a portion of the energy from the combusted fluid to power the compressor with the result being thrust. These two processes create very interesting and complicated fluid mechanics, which have recently been simulated using the RANS formulation. In particular, the three-dimensional "rotor-stator" combination has been simulated for a typical turbine configuration by Rai [18]. Again in this application a zonal grid system 
was used to model the complex geometry. Because of the inherently unsteady nature of this problem, the zonal grid system was constructed with an interface surface such that the rotor grid can move relative to the stator grid.

Typical results for a three-dimensional turbine application are displayed in Figs. 7 and 8 for an inlet Mach number of 0.07. Again, this simulation used the standard Baldwin-Lomax turbulence model [15]. In this computation two blades associated with one turbine stage, one rotor and one stator; the inner hub and the outer shroud; and the clearance between the rotor tip and the shroud are all simulated. Multiple rotor and stator blades within the same stage are simulated via periodicity boundary conditions. Figure 7 shows the time-averaged limiting streamlines on the stator pressure side and the rotor suction side. Note the confluence of flow at about $70 \%$ of span on the rotor and the flow distribution on the rotor tip. Figure 8 shows timeaveraged pressures on the stator and rotor at mid span, both the pressure and suction surfaces The computed results are compared with experiment and are in excellent agreement. Unsteady pressures are also compared with experiment in [18] and are in good agreement with experiment.

The rotor-stator simulation just discussed is similar to the first two RANS simulations in many ways but differs in one important way -- rotor-stator flows are inherently unsteady. This puts a different light on some of the computational statistics. Because of the requirement for time accuracy, the integration time or number of iterations for this calculation is larger and requires on the order of 100 hours on a single-processor Cray 2 time. The grid used in this simulation involved 200,000 points distributed in five zones. Thus, the data base associated with either a single time step or a time-averaged solution is about 1.6 million words. However, the data base associated with one cycle, which is the time it takes for the rotor to move from one stator to the next stator, is about 3.2 billion words! Even though the entire time-dependent solution does not

STATOR PRESSURE SIDE / ROTOR SUCTION SIDE

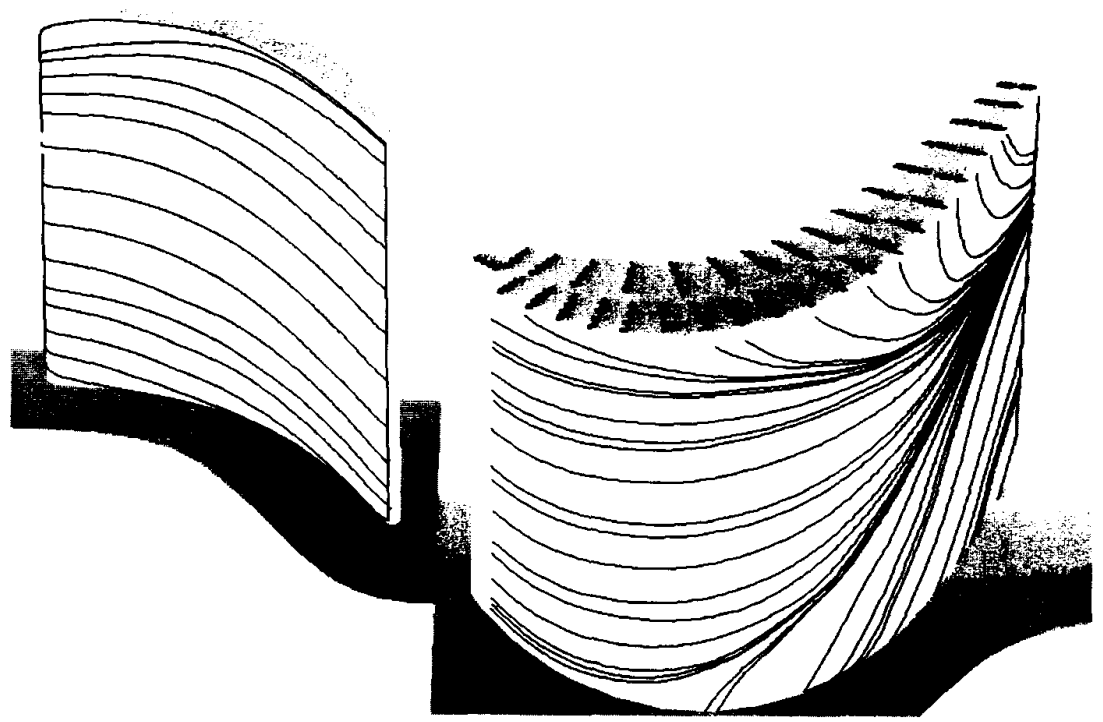

Figure 7. Computed time-averaged limiting streamlines on the pressure side of the stator and the suction side of the rotor, Mach $=0.07$, Rai [18]. 


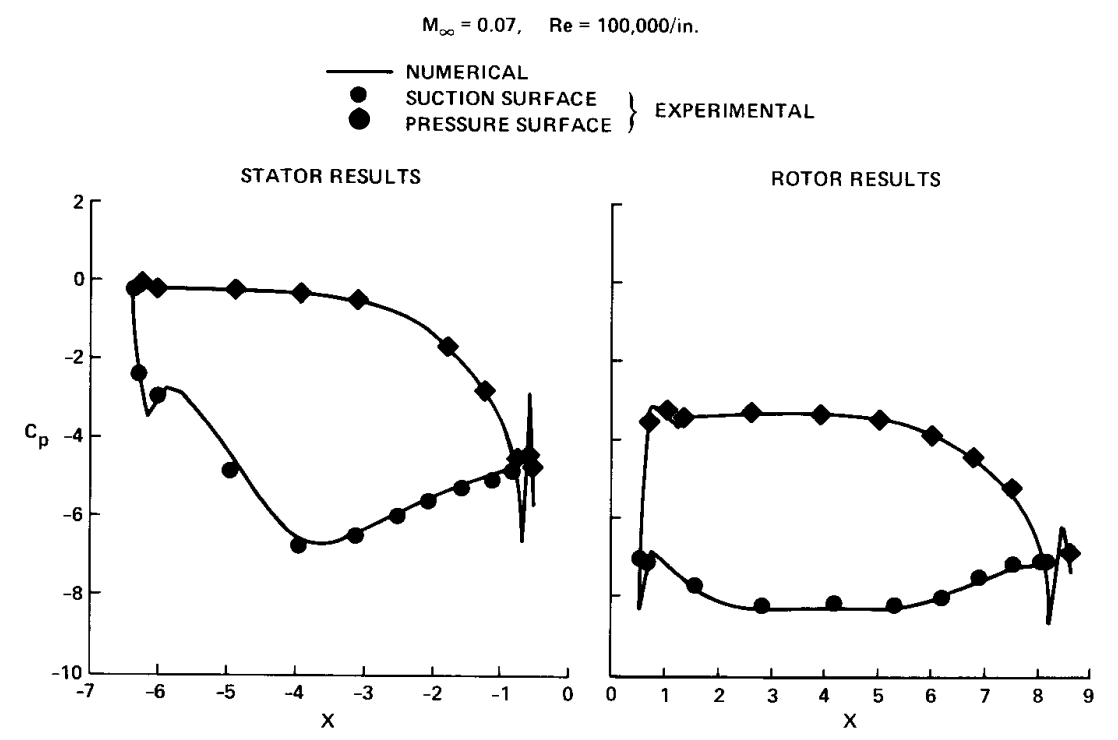

Figure 8. Computed and experimental time-averaged pressures on the stator and rotor blades at midspan, Mach $=0.07$, Rai [18].

have to be in main memory at one time, this is the solution size that has to be retained, which is a significant limiting factor for unsteady CFD applications. In addition, the only possible way to understand the full extent of the fluid physics contained in such a solution is through computer graphics. The manipulation of such a large data base for graphical display is another significant problem facing the CFD field. However, with recent and anticipated advances in high-speed graphical work stations, this problem area is currently receiving considerable attention and should allow for the dramatic display of future CFD results.

\section{CONCLUDING REMARKS}

The field of Computational Fluid Dynamics has been briefly reviewed with particular emphasis on the Reynolds-averaged Navier-Stokes formulation. Several three-dimensional applications have been presented and discussed to highlight this review. The computational requirements, both execution speed and memory, needed to meet the needs of these applications have also been presented and discussed. Although these requirements are demanding, especially for the more complete formulations and more complex applications, significant progress and utilization of this technology across a broad front of applications is forcast for the next decade.

\section{REFERENCES}

1. Holst, T. L., Slooff, J. W., Yosihara, H., and Ballhaus, Jr., W.F., "Applied Computational Transonic Aerodynamics," AGARDograph No. 266, Aug. 1982.

2. Rogallo, R. S. and Moin, P., "Numerical Simulation of Turbulent Flows," Annual Review of Fluid Mechanics. Vol. 16, 1984, pp. 99-137. 
3. Holst, T. L., "Numerical Solution of the Navier-Stokes Equations About Three-Dimensional Configurations--A Survey," Presented at the NASA Conference for Supercomputing in Aerospace, March 10-12, 1987, NASA CP-2454, pp. 281-298.

4. Peterson, V. and Arnold, J., "The Impact of Supercomputers on Experimentation: A View from a National Laboratory," ÁSEE Annual Conference Proceedings, 1985, pp. 1388-1401.

5. Anderson, D. A., Tannehill, J. C., and Pletcher, R. H., Computational Fluid Mech.and Heat Transfer, Hemisphere Publishing Corp., Washington, 1984.

6. Peyret, R. and Viviand, H., "Computation of Viscous Compressible Flows Based on the Navier-Stokes Equations," AGARDograph No. 212, 1975.

7. Chapman, D. R., "Computational Aerodynamics--Development andOutlook," AIAA J, Vol. 17, No. 12, Dec. 1979, pp. 1293-1313.

8. Chapman, D. R., "Trends and Pacing Items in Computational Aerodynamics," Lecture Notes in Physics, Vol. 141, Eds. Reynolds, W. C. and MacCormack, R., Springer-Verlag, Berlin Heidelberg, Germany, 1981.

9. Kutler, P., "A Perspective of Theoretical and Applied Computational Fluid Dynamics," AIAA J, Vol. 23, No. 3, Mar. 1985, pp. 328-341.

10. Kutler, P., Steger, J. L., and Bailey, F. R., "Status of Computational Fluid Dynamics in the United States," AIAA Paper No. 87-1135-CP, June 1987.

11. Reznick, S. G. and Flores, J., "Strake Generated Vortex Interactions for a Fighter-Like Configuration," AIAA Paper No. 87-0589, Jan. 1987.

12. Flores, J., Reznick, S. G., Holst, T.L., and Gundy, K., "Transonic Navier-Stokes Solutions for a Fighter-Like Configuration," AIAA Paper No. 87-0032, Jan. 1987.

13. Flores, J., Chaderjian, N. M., and Sorenson, R. L., "Simulation of Transonic Viscous Flow over a Fighter-Like Configuration Including Inlet," AIAA Paper No. 87-1199, June 1987.

14. Flores, J. and Chaderjian, N. M., "The Numerical Simulation of Transonic Separated Flow about the Complete F-16A," AIAA Paper No. 88-2506, June 1988.

15. Baldwin, B. S. and Lomax, $H_{\text {., }}$ "Thin Layer Approximation and Algebraic Model for Separated Turbulent Flow," AIAA Paper No. 78-257, Jan. 1978.

16. Chaderjian, N. and Flores, J., Private communication, Oct. 1988.

17. NASA Ames Space Shuttle Flow Simulation Group, Buning, P. G., Chiu, I. T., Obayashi S., Rizk, Y. M., and Steger, J. L., "Numerical Simulation of the Integrated Space Shuttle Vehicle in Ascent," AIAA Paper No. 88-4359, Aug. 1988.

18. Rai, M., "Unsteady Three-Dimensional Navier-Stokes Simulations of Turbine Rotor-Stator Interaction," AIAA Paper No. 87-2058, June 1987. 